A. Sibille*, P. Van Bleyenbergh*, K. Lagrou ${ }^{\#}$, A. Verstraeten and W. Wuyts*

Depts of *Pneumology, and "Microbiology, University Hospitals Leuven, Leuven, and 'Dept of Pneumology, AZ Sint-Lucas, Ghent, Belgium.

Correspondence: W. Wuyts, University Hospital of Leuven, Dept of Pneumology, Herestraat 49, 3000 Leuven, Belgium. Email: wim.wuyts@uzleuven.be

Statement of Interest: None declared.

\section{REFERENCES}

1 Kauffman CA. Histoplasmosis. Clin Chest Med 2009; 30: 217-225, v.
2 Mukhopadhyay S, Gal AA. Granulomatous lung disease: an approach to the differential diagnosis. Arch Pathol Lab Med 2010; 134: 667-690.

3 Kauffman CA. Histoplasmosis: a clinical and laboratory update. Clin Microbiol Rev 2007; 20: 115-132.

4 Wheat LJ. Improvements in diagnosis of histoplasmosis. Expert Opin Biol Ther 2006; 6: 1207-1221.

5 Puckett TF. Pulmonary histoplasmosis; a study of twenty-two cases with identification of $H$. capsulatum in resected lesions. Am Rev Tuberc 1953; 67: 453-476.

6 El-Zammar OA, Katzenstein AL. Pathological diagnosis of granulomatous lung disease: a review. Histopathology 2007; 50: 289-310.

7 Wheat LJ, Freifeld AG, Kleiman MB, et al. Clinical practice guidelines for the management of patients with histoplasmosis: 2007 update by the Infectious Diseases Society of America. Clin Infect Dis 2007; 45: 807-825.

\title{
An unusual presentation of sarcoidosis with tetraplegia and severe osteolytic bone lesions
}

\section{To the Editors:}

Sarcoidosis is a granulomatous multisystem disorder of unknown aetiology. Pathology is characterised by non-necrotising granulomas, which may affect virtually any organ, most commonly the lungs, lymph nodes, skin and eyes. Lytic foci in the phalanges of the hands and feet are common, whereas it is rare to see a widespread effect to the bone.

We present a case of severe diffuse osteolytic bone involvement and tetraplegia presenting as the first manifestation of sarcoidosis.

A 54-yr-old male was admitted to hospital after a bike accident, presenting with total paralysis of his left arm and paresis of his right arm and both legs. Computed tomography (CT) revealed fracture of C2 and widespread osteolytic lesions in the vertebrae, sternum, clavicles, skull, costae and pulmonal nodular infiltrates. The patient had no respiratory complaints.

Positron emission tomography with ${ }^{18}$ F-fluoro-2-deoxy-Dglucose (FDG-PET)/CT showed areas of pathological uptake that would suggest malignancy, but biopsy from the bone marrow showed non-necrotising granulomas compatible with sarcoidosis. The suspicion of cancer was maintained due to the rareness of osteolytic sarcoidosis, but repeated biopsies from os ileum and os parietale confirmed the diagnosis of sarcoidosis, and prednisolone and methotrexate were initiated. A stabilising operation of the cervical spine was performed; 2 months later, another operation with laminectomy at C1 level was necessary for decompression of the spinal cord.

At the time of diagnosis, pulmonary function tests showed a forced expiratory volume in $1 \mathrm{~s}$ (FEV1) of $1.8 \mathrm{~L}(43 \%$ of predicted), rising to $2.9 \mathrm{~L}$ (69\% pred) 6 months later. FEV1/ forced vital capacity (FVC) was normal; total lung capacity (74\% pred) and diffusion capacity (76\% pred) were both slightly decreased and did not improve with treatment.
Interleukin (IL)-2 receptor was elevated to $1,180 \mathrm{kU} \cdot \mathrm{L}^{-1}$ (normal range $223-710 \mathrm{kU} \cdot \mathrm{L}^{-1}$ ), whereas angiotensineconverting enzyme (ACE) was normal. There was a light hypercalcaemia $\left(1.43 \mathrm{mmol} \cdot \mathrm{L}^{-1}\right)$, slightly elevated C-reactive protein $\left(134 \mathrm{nmol} \cdot \mathrm{L}^{-1}\right)$ and a mild normochrome, normocytic anaemia $\left(7.5 \mathrm{mmol} \cdot \mathrm{L}^{-1}\right)$. These parameters were normalised after a few months of treatment.

Response to treatment was monitored with serum ACE, IL-2 receptor and bone scintigraphy. FDG-PET/CT and bone scintigraphy were performed early in the course of disease, and showed excellent correspondence (figs 1 and 2). Bone scintigraphy was chosen for monitoring.

Remission of the scintigraphic bone manifestations began after more than 1 yr of treatment, and was almost complete after 2 yrs. IL-2 receptor fell to normal levels within 2 months.

Prednisolone was gradually tapered and finally stopped after nearly 2 yrs of treatment. Immunosuppressive treatment was completed with intravenous biphosphonate (zoledronic acid), administered yearly for $3 \mathrm{yrs}$, and daily supplementation of calcium and vitamin D.

The patient underwent intensive rehabilitation after the operation with very satisfactory results. After 3 months, he was able to walk again, and he resumed work 2 yrs after the diagnosis. At the time of writing, the patient was still taking methotrexate and the disease was well controlled; methotrexate treatment was planned to be ceased during the coming months.

Bone involvement is frequent in sarcoidosis, and is seen in up to $13 \%$ of patients [1]. It is usually a late manifestation and is often associated with chronic pulmonary, cutaneous or multivisceral sarcoidosis. The most common manifestations are asymptomatic lytic lesions of the phalanges of the hands and feet [2]. Involvement of the vertebral column, the skull and the 


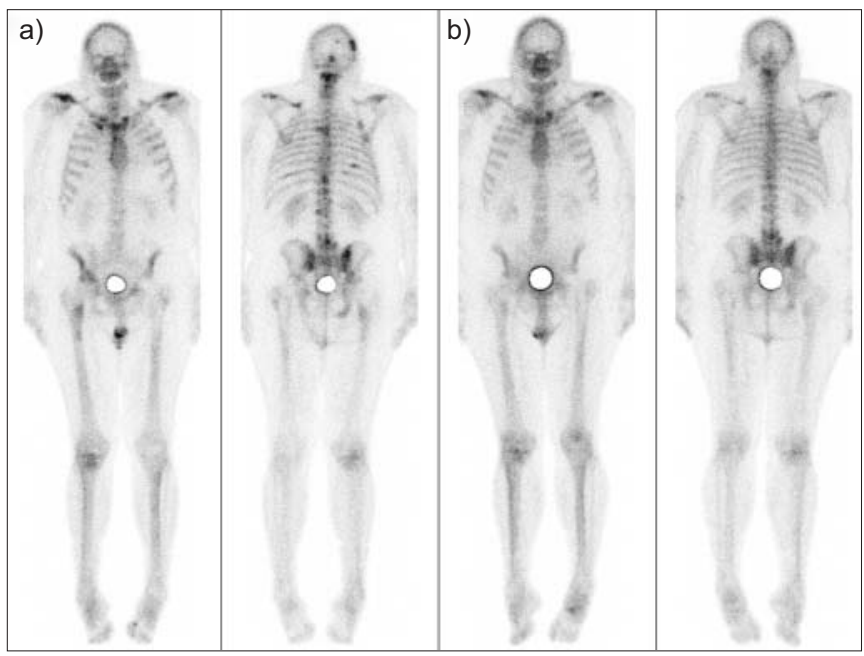

FIGURE 1. Bone scintigraphy showing a) multifocal increased uptake and b) marked regression after several months of treatment. a) Increased uptake in os parietale, os occipitale, both clavicles, both scapulae and the manubrium part of the sternum. Costae 8-9, C1-C7, Th4-5, Th10-11, L2 and L5. In the pelvis, increased uptake is seen in the right corpus ossis ilii, the right femur and the right caput tibiae. b) Increased activity in the cervical spine, L5 and os parietale dxt but complete regression of other manifestations.

sternum is rare, and virtually all publications are case reports. A recent review presented $<50$ cases of vertebral sarcoidosis [3] and only nine of these cases had cervical involvement. To our knowledge, the associated involvement of the skull, sternum and spine with tetraplegia as the presenting symptom has not previously been reported.
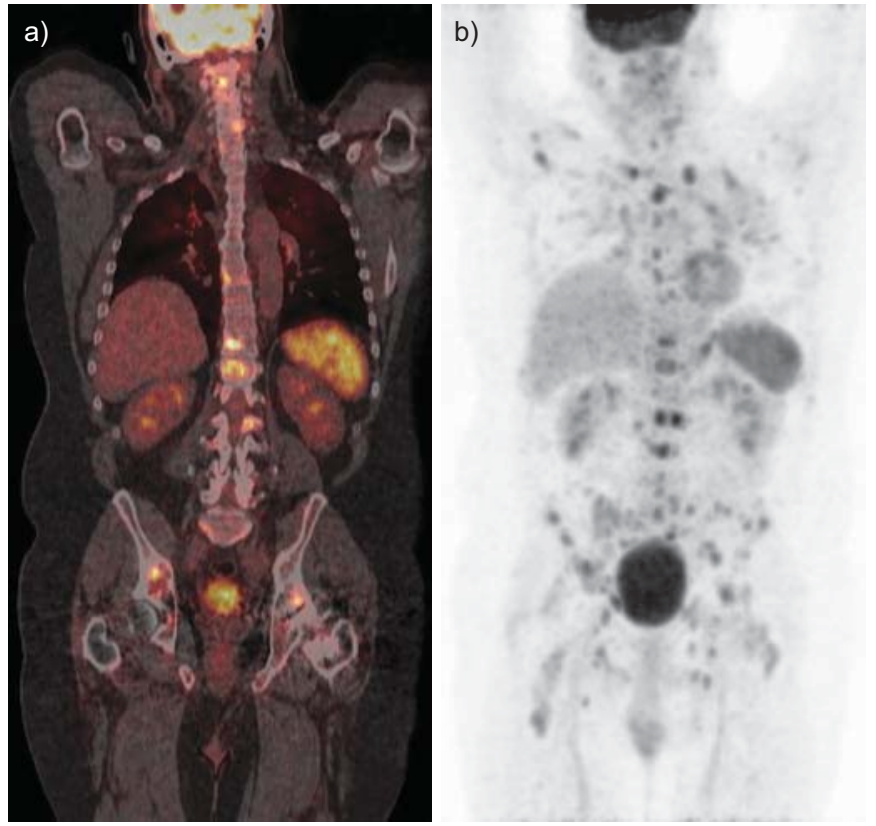

FIGURE 2. a) Positron emission tomography with ${ }^{18} \mathrm{~F}$-fluoro-2-deoxy-Dglucose (FDG-PET) and b) fused PET/computed tomography showing multiple areas of increased uptake in the bones, lungs, mediastinal lymph nodes, spleen, abdominal lymph nodes and heart. Magnetic resonance imaging of the heart and echocardiography performed afterwards were normal; suspicion of cardiac involvement was not confirmed.
Treatment of sarcoid bone involvement is anecdotal. In several cases, treatment with prednisolone has led to symptom remission and to objective remission of the condition's affects [4-6], whilst other cases have been noted where there has been no treatment and no evidence of progression [7]. Successful treatment of vertebral sarcoidosis with methotrexate has also been previously reported [8]; in general though, treatment of bone sarcoidosis is disappointing [9].

Treatment with corticosteroids implies risk of osteoporosis and pathological fractures, and the role of calcium and vitamin D in sarcoidosis is controversial. Careful prevention of osteoporosis and monitoring of calcium metabolism is especially important in vertebral sarcoidosis. Biphosphonates as prevention and treatment of corticosteroid-induced osteoporosis in sarcoidosis are effective [10], but no recommendations exist for biphosphonate therapy in bone manifestations of sarcoidosis.

Bone scintigraphy is helpful in patients with known or suspected osseous sarcoidosis, in order to identify the extent of osseous disease activity and to detect clinically silent areas that may be accessible for biopsy. It can detect clinically silent areas that may be accessible for biopsy, but does not provide information on visceral involvement in the same way as PET/CT.

$\mathrm{PET} / \mathrm{CT}$ and bone scintigraphy, as well as magnetic resonance imaging, are sensitive but they are not specific in sarcoidosis. Histological confirmation is necessary to establish diagnosis and exclude differential diagnoses, such as metastatic disease as in the case presented here.

Although osteolytic lesions are much more common in malignant diseases, rare presentations of non-malignant diseases such as sarcoidosis should be considered if malignancy is not proven within a reasonable time.

The present case demonstrates that treatment with immunosuppressives and intravenous biphosphonate is of major importance in the treatment of osteolytic sarcoidosis, and that PET/CT and bone scintigraphy are valuable in determining the extent of disease and monitoring the response to treatment.

\section{Hyldgaard*, E. Bendstrup*, O. Hilberg*, K. Hjorthaug ${ }^{\#}$ and M. Lovgreen*}

Depts of *Respiratory Medicine and \# Nuclear Medicine, Aarhus University Hospital, Aarhus, Denmark.

Correspondence: C. Hyldgaard, Aarhus University Hospital, Dept of Respiratory Medicine, Norrebrogade 44, Aarhus, 8000 C, Denmark. E-mail: charhyld@rm.dk

Statement of Interest: None declared.

\section{REFERENCES}

1 Neville E, Carstairs LS, James DG. Sarcoidosis of bone. QI Med 1977; 46: 215-227.

2 Sartoris DJ, Resnick D, Resnik C, et al. Musculoskeletal manifestations of sarcoidosis. Semin Roentgenol 1985; 4: 376-386.

3 Wendling D, Desmurs $\mathrm{H}$, Royer $\mathrm{F}$, et al. Inaugural cervical vertebral sarcoidosis. J Rheumatol 2008; 35: 362-365. 
4 Mangino D, Stover DE. Sarcoidosis presenting as metastatic bony disease. A case report and review of the literature on vertebral body sarcoidosis. Respiration 2004; 71: 292-294.

5 Talmi D, Smith S, Mulligan M. Central skeletal sarcoidosis mimicking metastatic disease. Skeletal Radiol 2008; 37: 757-761.

6 Binicer O, Sari I, Sen G, et al. Axial sarcoidosis mimicking radiographic sacroiliitis. Rheumatol Int 2009; 29: 343-345.

7 Aberg C, Ponzo F, Raphael B, et al. FDG positron emission tomography of bone involvement in sarcoidosis. AJR Am J Roentgenol 2004; 182: 975-977.
8 Mana J, Gomez-Vaquero C, Dorca J, et al. Vertebral and rib sarcoidosis: long-term clinical remission with methotrexate. Clin Rheumatol 1999; 18: 492-494.

9 Wilcox A, Bharadwaj P, Sharma OP. Bone sarcoidosis. Curr Opin Rheumatol 2000; 12: 321-330.

10 Zisman DA, Shorr AF, Lynch JP 3rd. Sarcoidosis involving the musculoskeletal system. Semin Respir Crit Care Med 2002; 23: 555-570.

\section{Nodular pulmonary ossifications in differential diagnosis of solitary pulmonary nodules}

\section{To the Editors:}

When considering a solitary pulmonary nodule or coin lesion, the differential diagnosis comprises a lot of different disease entities. Although uncommon, in specific patient groups, pulmonary ossifications should also be considered. We report two patients in whom the diagnosis of pulmonary ossification was confirmed pathologically and present a general discussion on this subject.

First, in a 68-yr-old patient with a history of hypercholesterolaemia and hypertension, a suspicious right mediastinal shadow was incidentally found on chest radiography after insertion of a central venous line. Personal history revealed poliomyelitis, retinal detachment and, especially, ventricular flutter for which he was resuscitated. Afterwards, he suffered from memory disturbances and even a frontal syndrome. A cardioverter-defibrillator was implanted. Chest radiography revealed a nodular aspect in the right lung hilum, which was not seen on a previous radiogram. Subsequent chest computed tomography (CT) demonstrated enlarged lymph nodes but no apparent ossifications. The mediastinum showed high metabolic activity on positron emission tomographic scan. Transbronchial biopsy was not conclusive. Cervical mediastinoscopy was negative. Subsequently, the right hilum was explored by thoracotomy. Enlarged lymph nodes were discovered and a wedge resection of the right middle lobe was performed to remove a calcified nodule of $\sim 1 \mathrm{~cm}$, surrounded by other small calcified lesions. Pathology demonstrated metaplastic bone tissue in the alveolar spaces and cores of ossification were diffusely spread across the pulmonary parenchyma. Diagnosis of diffuse nodular pulmonary ossification was made. Signs of pulmonary hypertension, such as intimal proliferation and focal myxoid degeneration of the parenchymal blood vessels were also present. Lymph nodes showed anthracosis and sarcoid reaction, but no signs of malignancy.

Secondly, a 71-yr-old patient with arterial hypertension and hypercholesterolaemia was diagnosed with a pT4N2M1 rectal carcinoma with liver metastases, for which he was treated by induction chemoradiation followed by rectal resection. After chemotherapy, a complete response of the liver metastases was initially achieved, but recurrent disease was diagnosed 1 yr later.
A wedge resection of two liver segments (II and VII) with cholecystectomy were performed followed by insertion of an intrahepatic PORT-A-CATH ${ }_{\circledast}$ system (Smiths Medical, Zaventem, Belgium). Pulmonary emboli occurred as postoperative complications. 6 months later, two other liver segments were resected (V and VI) and a further 3 months later, a right hepatectomy needed to be performed.

After diagnosis of two nodules in the right upper lobe suspicious for lung metastases 6 months after the hepatectomy, the patient was included in a phase II clinical trial investigating the effect of peroperative isolated lung perfusion with high-dose melphalan on lung metastases. The procedure was performed by an anterolateral muscle-sparing thoracotomy and, after lung perfusion, wedge excision of the suspicious nodules was performed. Pathology showed a metastasis of the colorectal carcinoma in one resection specimen and cores of ossification in the other, compatible with diffuse nodular pulmonary ossification. Remarkably, bone marrow was found in these ossification cores (fig. 1). All lymph nodes dissected during the procedure showed no signs of malignancy.

No post-operative complications were noted. Follow-up after 3 months revealed an uneventful course, with some tenderness remaining at the incision site.

Pulmonary ossification (PO) is an unusual condition where bone formation occurs within the parenchyma of the lung. Mostly, it is only recognised post mortem [1]. Ante mortem, it may be diagnosed radiographically [2] or after lung tumour resection, which is the case in our two patients. These POs can be spread diffusely in the lung, and may be divided into a nodular PO (NPO) and a dendritic PO (DPO), depending on their appearance on CT. Differentiation between DPO and NPO is presented in table 1 [1].

DPOs represent the less common form and are associated with pulmonary inflammation, pulmonary fibrosis, chronic obstructive pulmonary disease, asbestosis and pneumonia or may be idiopathic [3]. In a total of 1,393 adult autopsy cases performed by LARA et al. [1], only eight were identified as having dendritic calcifications, an average of $0.6 \%$ of all adult autopsies or one case every 3 yrs. The mechanisms behind the pathogenesis of $\mathrm{PO}$ still need further investigation. 